\title{
Differences in sustained attention capacity as a function of aerobic fitness
}

Antonio Luque-Casado ${ }^{1,2,3}$, Pandelis Perakakis ${ }^{1,4}$, Charles H. Hillman ${ }^{5}$, Shih-Chun Kao ${ }^{5}$, Francesc Llorens $^{6,7}$, Pedro Guerra ${ }^{1,4}, \&$ Daniel Sanabria ${ }^{1,2}$

1 Brain, Mind, \& Behavior Research Center. University of Granada, Spain.

2 Department of Experimental Psychology. University of Granada, Spain.

3 Department of Physical Education \& Sport. University of Granada, Spain.

4 Department of Personality, Evaluation \& Psychological Treatment. University of Granada, Spain.

5 Department of Kinesiology \& Community Health. University of Illinois at UrbanaChampaign, Illinois, USA.

6 Department of Physical Activity \& Sport Sciences. Catholic University of Valencia, Spain. 7 Universidad Internacional Valenciana (VIU), Valencia, Spain.

Corresponding author: Antonio Luque-Casado

Mailing address: Centro de Investigación Mente, Cerebro y Comportamiento (CIMCYC).

Office number 35 (343). Campus Universitario de Cartuja (s/n). 18071. Granada (Spain).

E-mail address: antonioluque@ugr.es 


\begin{abstract}
Purpose: We investigated the relationship between aerobic fitness and sustained attention capacity by comparing task performance and brain function, by means of event-related potentials (ERPs), in high- and low-fit young adults. Methods: Two groups of participants (22 higher-fit and 20 lower-fit) completed a 60' version of the Psychomotor Vigilance Task (PVT). Behavioral (i.e., reaction time; RT) and electrophysiological ERP (i.e., contingent negative variation; $\mathrm{CNV}$; and, P3) were obtained and analyzed as a function of time-on-task. A submaximal cardiorespiratory fitness test confirmed the between-groups difference in terms of aerobic fitness. Results: The results revealed shorter RT in higher-fit than in lowerfit participants in the first 36' of the task. This was accompanied by larger CNV amplitude in the same period of the task in higher-fit than in lower-fit group. Crucially, higher-fit participants maintained larger P3 amplitude throughout the task compared to lower-fit, who showed a reduction in the P3 magnitude over time. Conclusion: Higher fitness was related to neuroelectric activity suggestive of better overall sustained attention demonstrating a better ability to allocate attentional resources over time. Moreover, higher fitness was related to enhanced response preparation in the first part of the task. Taken together, the current dataset demonstrated a positive association between aerobic fitness, sustained attention, and response preparation.
\end{abstract}

Key words: Vigilance; ERP; physical activity; exercise; cognition; reaction time. 


\section{Introduction}

Over the past decades, growing evidence from various experimental approaches has shown that aerobic fitness and cognitive-behavioral performance are positively related (16). A major component of this research has revealed aerobic fitness-related improvements in a variety of tasks involving different cognitive functions, i.e., from processing speed to higherorder cognitive control or memory (34). Despite the progress on this topic, relatively little is known about an inherent cognitive process in the majority of these cognitive tasks that is necessary for optimal performance, i.e., sustained or vigilance attention. Here, we aimed at filling this gap by providing novel evidence of the positive relationship between aerobic fitness and the capacity to sustain attention (or to be vigilant) over time during task performance. To achieve this, we compared a higher-fit and a lower-fit group of young adults in terms of reaction time (RT) performance and brain function (by means of event-related potentials; ERPs) in a 60' long attentional task.

Sustained or vigilant attention is a higher-order cognitive function that determines the readiness to respond to relevant stimuli and the capacity to effectively allocate attentional resources over time. This cognitive function represents a fundamental component of the general cognitive capacities of humans since a reduced ability to monitor significant sources of information directly affects all cognitive abilities (i.e., slow responses and/or failures to respond to target stimuli [33]). In effect, the capacity to sustain attention is highly important both in specific laboratory contexts and in the completion of many everyday or professional activities that usually occur over long periods of time such as attending to academic lessons at school, driving, sports, surgery, or air traffic control (22). Crucially, our ability to sustain attention is far from stable and an extended period of attentional demands on a single task leads to a decrement in performance over time, which is known as time-on-task effect or 
vigilance decrement (8). Therefore, investigation into variables that might contribute against vigilance-related decrements in attention and performance over time is highly relevant.

Previous research has examined the relationship between aerobic fitness and attentional mechanisms (30), but a relatively scant literature to date has addressed the association between aerobic fitness and sustained attention in a direct manner like in the present study. For example, Bunce (6) evaluated the influence of physical fitness on age differences in vigilance as a function of the time course of a task and the level of task complexity, showing an attenuated vigilance decrement in higher-fit older adults in comparison to their lower-fit counterpart. Crucially, no group differences were found in young adults. The results of the few related studies testing children also point to a positive relationship between aerobic fitness and sustained attention. For instance, Pontifex, Scudder, Drollette, and Hillman (31) concluded that poor aerobic fitness was related to impaired vigilance on the basis of an observed increase in error of omissions and more sequences of omissions in lower-fit relative to higher-fit preadolescent participants during a flanker task. Chaddock et al. (7) investigated the time course of behavioral performance and brain functioning during a flanker task in preadolescents participants. They showed a decline in performance over time on incongruent trials, but only for lower-fit participants, who demonstrated a bilateral increase in activation in frontal and parietal brain regions from early to late blocks of trials. Higher-fit participants, in contrast to their lower-fit peers, showed decreased activity as a function of time-on-task, but greater activity was shown in early blocks with respect to lower-fit participants. Meanwhile, Ballester, Huertas, Yuste, Llorens, and Sanabria (2) showed a positive relationship between fitness and vigilance during adolescence, with higher-fit participants showing overall shorter RTs than lower-fit participants in the Psychomotor Vigilance Task (PVT). 
Despite a growing literature in children and some studies with older adults, the potential significant relationship between aerobic fitness and sustained attention in young adults remains poorly understood. Although cognitive health peaks during young adulthood (32), which could act by reducing the room for exercise-related improvement in cognitive function in this age group, research consistently demonstrates the importance of physical activity in keeping, and potentially improving, cognitive function throughout life (14). Additionally, the study of the relationship between sustained attention and aerobic fitness in this age range is highly relevant because of the disproportionate decline in physical activity from adolescence to early adulthood (21). To the best of our knowledge, the results reported by Luque-Casado, Zabala, Morales, Mateo-March, and Sanabria (27) represent the sole evidence of a selective association between aerobic fitness and sustained attention in young adults. This study showed better vigilance performance in higher-fit as compared to lower-fit participants, indexed by shorter overall RTs in a 10 minutes version of the PVT, while no differences where shown in an endogenous temporal orienting task and in a duration discrimination task. These results were taken as evidence suggesting superior sustained attention capacity in young higher-fit relative to lower-fit participants. Note, though, that Luque-Casado et al. reported group differences in overall RT but not in terms of the RT vigilance decrement.

Thus, convergent evidence suggests the important role of aerobic fitness on sustained attentional capacity but research is scarce and several important questions remain open. For example, the few studies to date assessing one of the factors that have been shown to tax sustained attention (i.e., the duration of the task [8]) as a function of aerobic fitness, have reported inconsistent results. A vigilance decrement of greater magnitude has been shown in lower-fit individuals relative to their higher-fit peers both in preadolescents and in older adults $(6,31)$, while no differences were found in young adults $(6,27)$. Importantly, previous 
studies have shown that prolonged sustained attention demands (i.e., 20 to 30 minutes) are needed to elicit a significant deterioration in sustained attention performance in young adults (13). Therefore, given that all the aforementioned studies used experimental tasks that typically last for only a few minutes (i.e., 10 minutes at the most), the use of a task with extended sustained attentional demands (i.e., exceeding 30 minutes of duration) may increase the likelihood of observing a between-groups difference in the magnitude of the time-on-task effect in young adults.

Additionally, the underlying neural basis of the aerobic fitness-related improvements in vigilance performance is another important issue that remains unknown. Two main ERP components of interest with regard to fitness and sustained attention are the P3 and the contingent negative variation (CNV). On the one hand, the $\mathrm{P} 3$ potential is commonly thought to reflect the amount of attentional resources directed toward task-relevant information in the stimulus environment (29). It has been one of the main indexes of interest in the study of sustained attention, elucidating a relationship between P3 amplitude and task performance over time (i.e., with RTs increasing and P3 amplitude decreasing [20]). Crucially, aerobic fitness has also been related to differences in P3 amplitude, with greater fitness related to larger P3 amplitude (15); thus, fitness may serve as a buffer against vigilance-related decrements in attention and performance over time. On the other hand, the CNV is a slow negative wave occurring during the preparatory interval between a warning signal and an impending stimulus that requires a response, which appears to reflect sensory, cognitive and motor preparation processes (37). Importantly, studies have reported a positive relationship between aerobic fitness and the magnitude of the $\mathrm{CNV}$, leading to improved cognitive performance in aerobically fit individuals compared with their lesser fit counterparts across several cognitive tasks assessing working memory (19), cognitive control (35) or processing speed (1). However, although the magnitude of the CNV has been shown to depend on 
sustained attention (4), the association between aerobic fitness and the magnitude of the CNV has not been investigated on the basis of sustained attentional performance.

Thus, as noted above, the present study stands to provide novel evidence of the relationship between aerobic fitness, behavioral performance and brain function of young adults in a prolonged sustained attention task during the pre-stimulus response preparation (i.e., CNV) and post-stimulus periods (i.e., P3). Based on previous evidence $(1,15,19,35)$, we expected the higher-fit group to have larger CNV and P3 amplitude values than the lowerfit group, which would also be related to higher overall performance in the vigilance task. Further, we predicted that both CNV and P3 amplitudes would gradually deteriorate as a result of the time-on-task effect, but with the higher-fit group showing an attenuated vigilance decrement and maintaining larger overall values during the course of the task.

\section{Method}

\section{Participants}

An a priori power analysis was conducted to determine the minimum sample size required for a power level of .80 . This analysis was based on data from the previous study by Luque-Casado et al. (27) where they compared performance in the PVT of a group of young cyclists and triathletes (higher-fit) to that of a group of young adults with sedentary lifestyle (lower-fit). This analysis gave an outcome of 22 participants per group.

We recruited 50 young male adults to participate in the present study, 25 undergraduate students from the University of Granada (Spain) to be included in the lower-fit group, and 25 young adults ( 15 members from triathlon local clubs and 10 from the Faculty of Physical Activity and Sport Sciences, University of Granada, Spain) to be included in the higher-fit group. The participants in the higher- and lower-fit group met the inclusion criteria of reporting at least 8 hours of training per week or less than 2 hours, respectively. Eight out of 50 participants (3 higher-fit and 5 lower-fit) were subsequently excluded from analyses 
(see data reduction section). Thus, only data from the remaining 42 participants are reported (see Table 1).

The experiment reported herein was conducted according to the ethical requirements of the local committee and complied with the ethical standards laid down in the 1964 Declaration of Helsinki. All participants gave informed consent prior to their inclusion in the study, had normal or corrected-to-normal vision, and no history of neuropsychological impairment. They were required to maintain a regular sleep-wake cycle for at least one day before the study and to avoid caffeine and vigorous physical activity before the laboratory visit. All participants' data were analyzed and reported anonymously.

Table 1. Mean and 95\% Confidence Interval (CI) of descriptive and fitness data for the higher-fit and lower-fit groups.

Higher-fit $\quad$ Lower-fit

\section{Anthropometrical characteristics}

Sample size ${ }^{\mathrm{a}}$

Age (years)

Height (cm)

Weight $(\mathrm{kg})$

Body Mass Index $\left(\mathrm{kg} \cdot\left(\mathrm{m}^{2}\right)^{-1}\right)$

Incremental test parameters

Time to VAT (s)

$\mathrm{VO}_{2}\left(\mathrm{~mL} \cdot \mathrm{min}^{-1} \cdot \mathrm{kg}^{-1}\right)$ at VAT

Relative power output at VAT $\left(\mathrm{W} \cdot \mathrm{kg}^{-1}\right)$
20

$23[22,24]$

$1.76[1.74,1.78]$

$1.78[1.75,1.81]$

$69.6[67.1,72.1]$

$76.9[69.0,85.6]$

$22.4[21.7,23.1]$

$24.1[22.1,26.2]$

\footnotetext{
${ }^{\mathrm{a}}$ Only data of the participants included in the analyses are reported. VAT (ventilatory anaerobic threshold).
} 


\section{Procedure}

Upon arrival to the laboratory, participants were seated in front of a computer in a dimly illuminated, sound attenuated room with a Faraday cage. First, participants signed the informed consent and were prepared for electrophysiological measurement. Participants then received verbal and written instructions regarding the PVT and practiced the task for one minute. The experiment consisted of a single 60 'block. Once they completed the PVT, all participants performed a submaximal incremental cycle-ergometer test to evaluate their fitness level. The experimental session was administered during daylight hours, with approximately half the participants participating in the morning (i.e., 11 athletes, 11 nonathletes) and the other half in the afternoon (i.e., 11 athletes, 9 non-athletes). The entire experimental session lasted $2 \mathrm{~h}$ approximately.

\section{Incremental effort test}

A brief preliminary anthropometric study of each participant was performed to measure height, weight and body mass index (BMI; see Table 1). We used a ViaSprint $150 \mathrm{P}$ cycle ergometer (Ergoline GmbH, Germany) to induce physical effort and to obtain power values, and a JAEGER Master Screen gas analyzer (CareFusion GmbH, Germany) to provide a measure of gas exchange during the effort test. Prior to the start of the test, participants were fitted with a Polar RS800 CX monitor (Polar Electro Öy, Kempele, Finland) to record their heart rate (HR) during the incremental exercise test and the cycle ergometer was set to the individual anthropometric characteristics.

We used a modified version of the incremental effort test from the previous study by Luque-Casado et al. (27). The incremental effort test started with a 3 min warm-up at $30 \mathrm{~W}$, with the power output increasing $10 \mathrm{~W}$ every minute. The test began at $60 \mathrm{~W}$ and was followed by an incremental protocol with the power load increasing $30 \mathrm{~W}$ every 3 minutes. Workload increased progressively during the third minute of each step ( $5 \mathrm{~W}$ every 10 
seconds); therefore, each step of the incremental protocol consisted of 2 minutes of stabilized load and one minute of progressive load increase. Each participant set his preferred cadence $\left(60-90 \mathrm{rev} \cdot \mathrm{min}^{-1}\right)$ during the warm-up. They were asked to maintain this cadence throughout the protocol. The ergometer software was programmed to increase the load automatically.

Determination of the ventilatory anaerobic threshold (VAT) was based on the respiratory exchange ratio (RER) $\left[\mathrm{RER}=\mathrm{CO}_{2}\right.$ production $/ \mathrm{O}_{2}$ consumption $]$. More specifically, VAT was defined as the $\mathrm{VO}_{2}$ at the time when RER exceeded the cut-off value of $1.0(9,40)$. The researcher knew that the participant had reached his VAT when the RER was equal to 1.00 and did not drop below that level during the 2 min constant load period or during the next load step, never reaching the 1.1 RER. The submaximal incremental test ended once the VAT was reached. The oxygen uptake (VO2 $\left.\mathrm{ml} \cdot \mathrm{min}^{-1} \cdot \mathrm{kg}^{-1}\right), \mathrm{RER}$, relative load $\left(\mathrm{W} \cdot \mathrm{Kg}^{-1}\right)$, heart rate $(\mathrm{bpm})$ and time of the test (s) were continuously recorded during the entire incremental test. The fitness level of the participants was determined from the dataset obtained during the incremental physical test (see Table 1).

\section{The Psychomotor Vigilance Task}

We used a PC with a 19' 'monitor and E-Prime software (Psychology Software Tools, Pittsburgh, PA, USA) to control the stimulus presentation, response collection, and to generate and send triggers indicating the condition of each trial for offline sorting, reduction, and analysis of EEG and behavioral data. The center of the PC screen was situated $\sim 60 \mathrm{~cm}$ from the participant's head and at eye level. The device used to collect responses was a PC keyboard.

The procedure of the PVT was based on the original version (39). This task was designed to measure vigilance by recording participants' RT to visual stimuli that occur at random inter-stimulus intervals $(3,39)$. Each trial began with the presentation of a blank screen in a black background for $2000 \mathrm{~ms}$ and subsequently, an empty red circumference 
$\left(6.68^{\circ} \times 7.82^{\circ}\right)$ appeared in a black background. Later, in a random time interval (between 2000 and $10000 \mathrm{~ms}$ ), the circumference was filled all at once in a red color. Participants were instructed to respond as fast as they could once they had detected the presentation of the filled circle. The filled circle was presented for $500 \mathrm{~ms}$ and the participants had a maximum of $1500 \mathrm{~ms}$ to respond. They had to respond with their dominant hand by pressing the space bar on the keyboard. A RT visual feedback message was displayed for $300 \mathrm{~ms}$ after response, except in case of an anticipated response ("wait for the target') or if no response was made within $1000 \mathrm{~ms}$ after target offset ("you did not answer"). Following the feedback message the next trial began. Response anticipations were considered errors. The task comprised a single block of 60 minutes of total duration and the mean number of trials per participant was $402 \pm 8.9$.

\section{Electroencephalogram (EEG) recordings}

Continuous EEG data were recorded using a BioSemi Active Two system (Biosemi, Amsterdam, Netherlands) and were digitized at a sample rate of $1024 \mathrm{~Hz}$ with 24-bit A/D conversion. The 64 active scalp $\mathrm{Ag} / \mathrm{AgCl}$ electrodes were arranged according to the international standard 10-20 system for electrode placement using a nylon head cap. The common mode sense (CMS) and driven right leg (DRL) electrodes served as the ground, and all scalp electrodes were referenced to the CMS during recording. The cap was adapted to the individual's head size and each electrode was filled with Signa Electro-Gel (Parker

Laboratories, Fairfield, NJ, USA) to optimize signal transduction. Participants were instructed to avoid eye movements, blinking and body movements as much as possible, and to keep their gaze on the center of the screen during task performance.

\section{Data reduction}

The behavioral data analyses were performed on the overall participants' mean RTs. Trials with RTs below $100 \mathrm{~ms}(0.03 \%)$, anticipations (i.e., responses prior to the target 
presentation; $1.49 \%$ ) and omissions (if no response was made within $1000 \mathrm{~ms}$ after target offset; $0.24 \%$ ) were discarded from the analysis (3).

We used a combination of bespoke Matlab scripts (Matlab 2013a, Mathworks Inc.), EEGLAB toolbox (version 13.2.2b, [10]) and ERPLAB toolbox (version 4.0.2.3, [23]) for processing and analyzing ERP data. Continuous data were down-sampled to $256 \mathrm{~Hz}$, merged offline with behavioral data and re-referenced to the average of all electrodes (average common reference). Noisy scalp electrodes were identified via visual inspection (only in 3 of the participants) and were replaced by an average of the voltages recorded at other neighbor scalp electrodes ( 3 electrodes on average were replaced in these subjects). We applied Independent Component Analysis (ICA; [10]) to correct eye blink artifacts (17). In order to remove baseline drifts, data were high-pass filtered $(0.1 \mathrm{~Hz} ; 12 \mathrm{~dB} /$ octave $)$ before running ICA. Prototypical ICA components representing eye movements and blinks were assessed on the raw EEG data before being excluded to corroborate their consistency and temporal match with the ocular artifacts. The ocular ICA components were removed in a systematic way for all participants to avoid any bias across the groups. On average, one independent component was removed per participant.

Once the ocular artifacts were corrected, separate epochs were constructed for cues (between -200 and $2000 \mathrm{~ms}$ relative to cue onset) and targets (between -200 and $1000 \mathrm{~ms}$ relative to target onset). The protocol typically employed to elicit the CNV is a two-stimulus (S1-S2) paradigm in which changes in amplitude between warning (S1) and imperative stimuli (S2) are measured. Note that $2000 \mathrm{~ms}$ is the minimum duration of the random interval between the cue and the target in the PVT paradigm and therefore, the point of maximal uncertainty. There is previous evidence showing a reliable CNV potential even under conditions of high uncertainty about the onset of S2 (36). Then, the PVT paradigm whereby participants have to respond to a target stimulus (S2) that appears in a random interval 
between 2 and 10 seconds after the presentation of a cue stimulus (S1) allows the measurement of the CNV. The $200 \mathrm{~ms}$ pre-stimulus period was used for baseline correction both in cues and targets epochs. Subsequently, data were filtered with a $30-\mathrm{Hz}$ low-pass cutoff ( $24 \mathrm{~dB}$ /octave). Remaining artifacts (EMG, noisy electrodes, etc.) exceeding $\pm 100 \mu \mathrm{V}$ in amplitude were detected and the epochs including those artifacts were excluded from further analysis. To ensure a sufficient signal-to-noise ratio and to reduce the possibility that the type I error rate was inflated by post hoc exclusion of subjects, we set an a priori criteria of excluding participants for whom more than $25 \%$ of trials were rejected $(23,26)$. This resulted in the exclusion of three higher-fit and five lower-fit participants. A minimum of 68 trials per condition was maintained. Separate grand average waveforms were constructed across all participants according to both cues and targets categories.

\section{Data measure and electrodes selection}

For cue and target analyses, amplitude was calculated as the mean voltage in a specified temporal window and electrodes site. The temporal windows were chosen on the basis of visual inspection of the grand average waveforms. The electrodes selection for both cue-locked and target-locked analyses was a two-stage process. First, several electrodes were selected for each potential of interest based on the topographical distribution of the scalp activity (see Figures 1 and 2). Next, electrodes for statistical analyses were chosen by their maximal positive or negative voltage value from each cluster, respectively. Thus, each potential was represented by an average of the selected electrodes. Specifically, the CNV potential was represented at frontal and central sites as the maximal negative mean amplitude between 1500 and 2000 ms after cue onset at electrode $\mathrm{Fz}, \mathrm{FCz}, \mathrm{Cz}$ and $\mathrm{CPz}$. The P3 potential was represented at posterior sites as the maximal mean amplitude between 240 and 440 ms after target onset at Pz and POz. 
Figure1. Grand average waveforms and topographic scalp distribution of the

Contingent negative Variation (CNV) as a function of Group and Block. Grand average waveforms are presented at Fz electrode. Time zero represents the cue stimulus appearance. Separate graphs for higher-fit (a) and lower-fit (b) are shown for clarity. Color lines are used to represent the waveforms as a function of Block. Gray marks show the time windows analyzed (i.e., 1500-2000 ms). Topographic scalp distribution of CNV amplitude (spectrum scale: blue to red) is illustrated for the higher-fit group (c) and lower-fit group (d) as a function of Block. The electrode sites included in the analyses are highlighted in bold in the topographic plots.

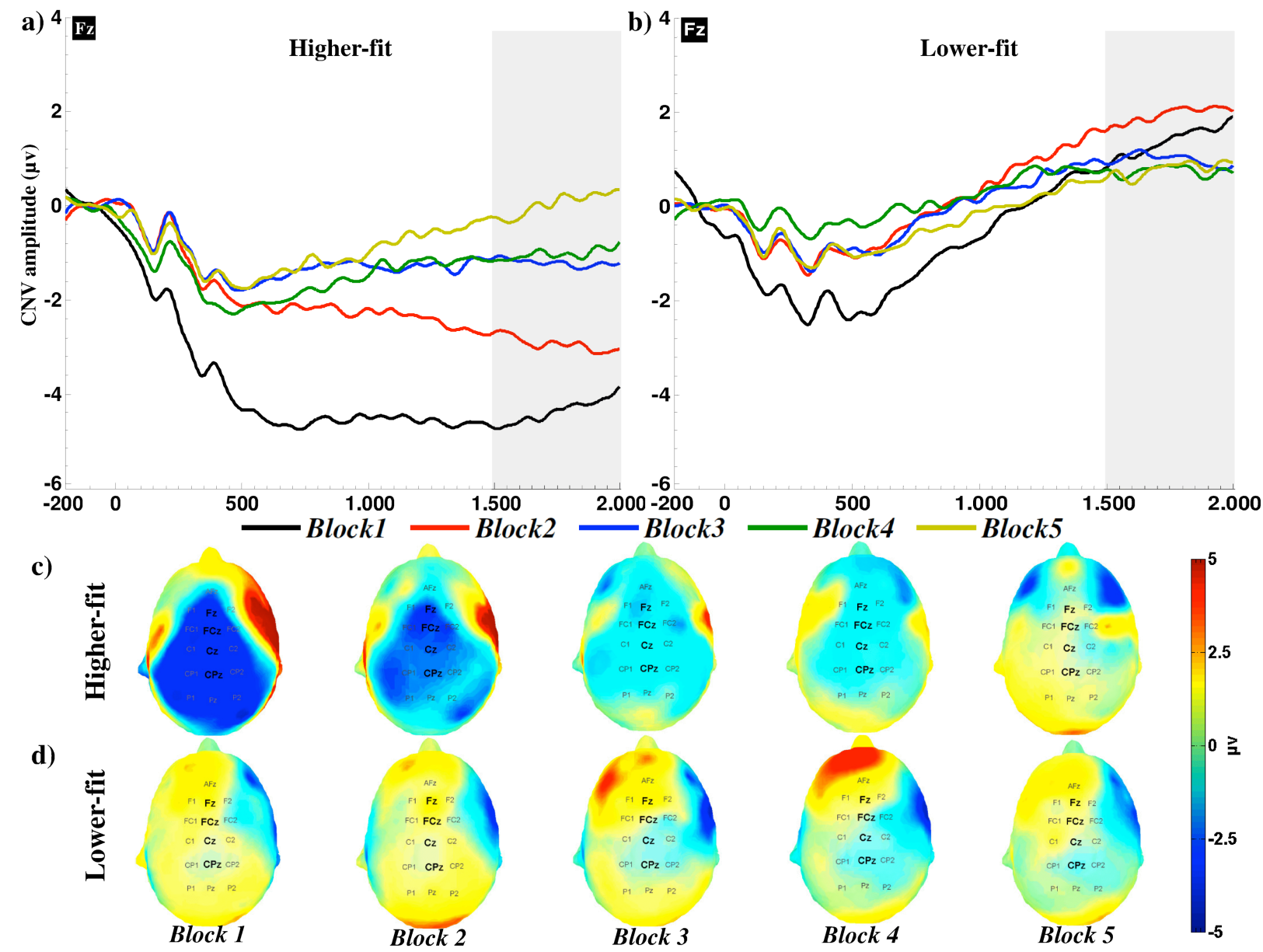


Figure2. Grand average waveforms and topographic scalp distribution of the P3

amplitude as a function of Group and Block. Grand average waveforms are presented at $\mathrm{Pz}$ electrode. Time zero represents the target stimulus appearance. Separate graphs for higher-fit (a) and lower-fit (b) are shown for clarity. Color lines are used to represent the waveforms as a function of Block. Gray marks show the time windows analyzed (i.e., 240-440 ms).

Topographic scalp distribution of P3 amplitude (spectrum scale: blue to red) is illustrated for the higher-fit group (c) and lower-fit group (d) as a function of Block. The electrode sites included in the analyses are highlighted in white in the topographic plots.

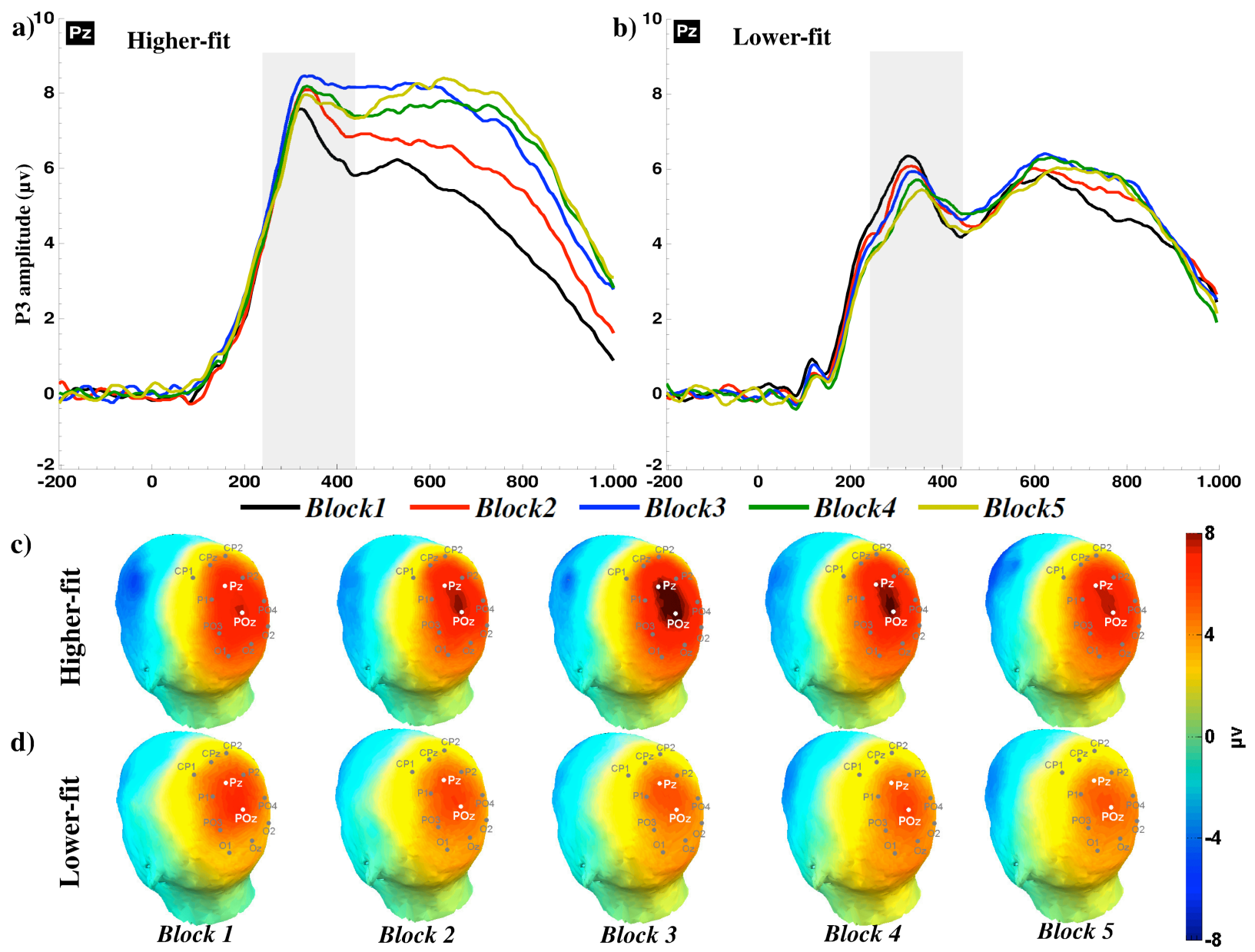

\section{Design and Statistical analysis}


Three sets of dependent variables were evaluated in this study: 1) Participants' descriptive and fitness data (i.e., anthropometrical and incremental exercise test parameters); 2) behavioral data (i.e., overall mean RTs); and 3) ERP data (i.e., CNV and P3 mean amplitude values). For the behavioral and ERP data, five temporal blocks of 12 minutes were considered for the analysis to measure the time-on-task effect.

Nonparametric permutation tests were used to analyze the data. Importantly, these tests are exact, unbiased and assumption-free in terms of the underlying distribution of the data $(11,28)$. We followed a general label exchange procedure for within-participants factorial designs (12) using a Monte Carlo approach.

The participants' descriptive and fitness data were analyzed using 1-way betweengroups design. For the behavioral and ERP data, we had a factorial design with the betweengroups variable of group (higher-fit and lower-fit) and the within-groups variable of time-ontask (block1, block2, block3, block4 and block5). Significant main effects and interactions were further explored by using post-hoc, pairwise comparisons and separate main effects analyses when appropriate. Multiple comparisons correction was accounted for by applying the false discovery rate (FDR) approach. 95\% confidence intervals (CI) and probability threshold values are reported.

\section{Results}

\section{Descriptive and fitness data}

The permutations tests for independent samples revealed significant differences between groups in all the incremental test parameters (i.e., time to VAT (s), relative power

output $\left(\mathrm{W} \cdot \mathrm{kg}^{-1}\right)$ at VAT and $\mathrm{VO}_{2}\left(\mathrm{~mL} \cdot \mathrm{min}^{-1} \cdot \mathrm{kg}^{-1}\right)$ at VAT) $($ all $p s<.01)$. All data showed evidence of the difference in fitness level between groups (see Table 1). There were no statistically significant differences between groups in any of the anthropometrical parameters (all $p s \geq .11$ ). 


\section{Behavioral results}

Participants' mean RTs results showed significant main effects of group $(p<.01)$ and time-on-task $(p<.01)$. Crucially, both main effects were better qualified by the significant interaction between group and time-on-task ( $p<.01$; see Figure 3). Pairwise comparisons (FDR corrected; $p$-threshold $=.005)$ were performed between the higher-fit and lower-fit group within each temporal block. The comparisons showed significant differences between groups at block 1, 2 and 3 (all $p s \leq .005$ ) with higher-fit being faster than lower-fit group (see Figure 3). There were no significant differences when comparing both groups in the remaining blocks (all $p s \geq .78$ ).

Figure3. Mean and 95\% confidence interval of the response time (ms) as a function of Group and Block. * indicate significant differences between groups within each block $(p \leq .005)$.

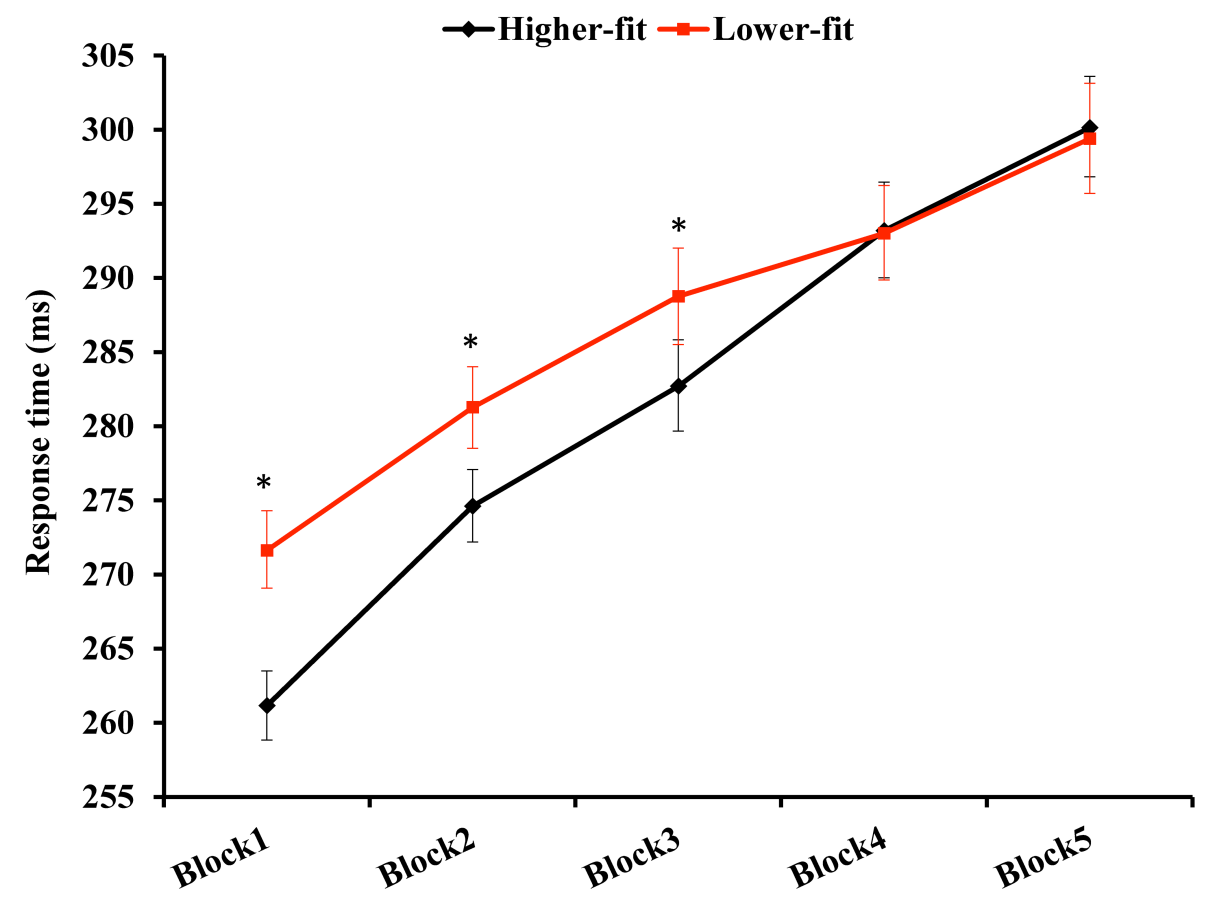

Electrophysiological results

Cue-locked ERPs

The CNV mean amplitude analyses revealed significant main effects of group $(p<.01)$ and time-on-task $(p<.01)$ that were better qualified by the significant interaction between group and time-on-task $(p<.01$; see Figure 1 and 4$)$. Further analyses showed a statistically 
significant main effect of time-on-task in the higher-fit group $(p<.01)$, with the amplitude of the CNV becoming less negative as time went on. However, this same analysis was not significant for the lower-fit group $(p=.19)$. Furthermore, pairwise comparisons (FDR corrected; $p$-threshold=.029) showed significant differences between groups at block 1 $(p<.01)$, block $2(\mathrm{p}<.01)$ and block $3(\mathrm{p}=.029)$. In all cases, the higher-fit group showed greater CNV negativity than lower-fit group (see Figure 4). There were no significant differences when comparing groups in blocks 4 and 5 (both $p s \geq .08$ ).

Figure4. Mean amplitude and 95\% confidence interval of the contingent negative variation $(\mathrm{CNV})$ as a function of Group and Block. * indicate significant differences between groups within each block $(p \leq .03)$.

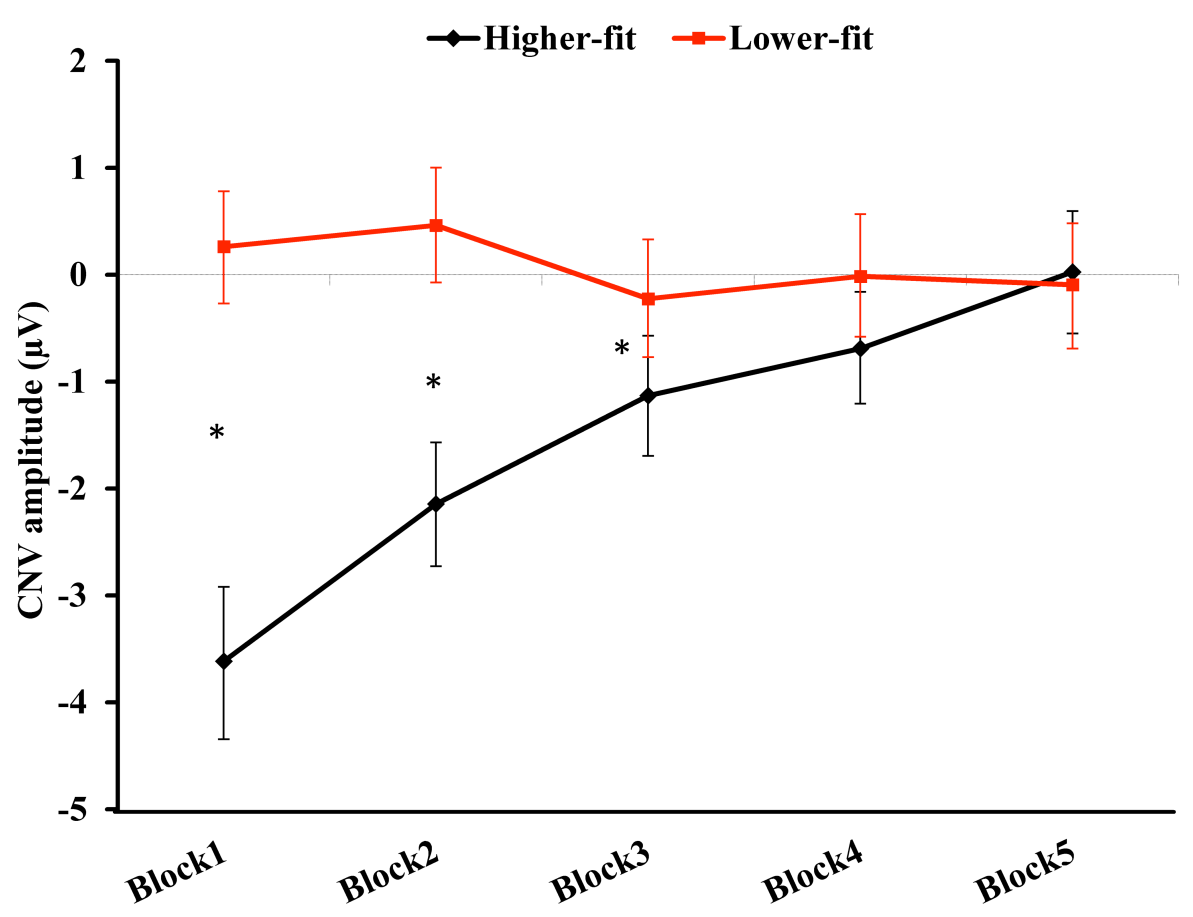

Target-locked ERPS

The P3 mean amplitude results showed significant main effects of group $(p<.01)$ and time-on-task $(p<.01)$. Again, the interaction between group and time-on-task reached statistical significance $(p<.01$; see Figure 2 and 5). Separate main effect analyses of time-ontask reached significance both for the higher-fit and lower-fit group (both $p s<.01$ ). In order to explain this interaction further we performed post-hoc comparisons (FDR corrected; $p$ - 
threshold=.003). For the higher-fit group, P3 amplitude values peaked in block 3 . There were statistically significant differences when comparing block 3 with respect to blocks 1,2 and 5 (all $p s \leq .003$ ). Additionally, the comparison between blocks 1 and 4 also showed significant differences $(p<.001)$. There were no significant differences when comparing the remaining blocks (all $p s \geq .06$; see Figure 5). In the case of the lower-fit group, the comparisons showed significant differences only between blocks 1 and 5 ( $p=.003)$, with decreasing amplitude over time. None of the remaining comparisons between blocks reached statistically significant differences (all $p s \geq .02$; see Figure 5). Additionally, pairwise comparisons (FDR corrected; $p$ threshold $=.0001)$ showed significant differences between groups in all blocks (all $p s \leq .0001)$, with the higher-fit group showing greater P3 mean amplitude than the lower-fit group (see Figure 5).

Figure5. Mean amplitude and 95\% confidence interval of the P3 as a function of Group and Block. * indicate significant differences between groups within each block $(p \leq .0001) . \dagger$ indicate significant differences between blocks within each group $(p \leq .003)$.

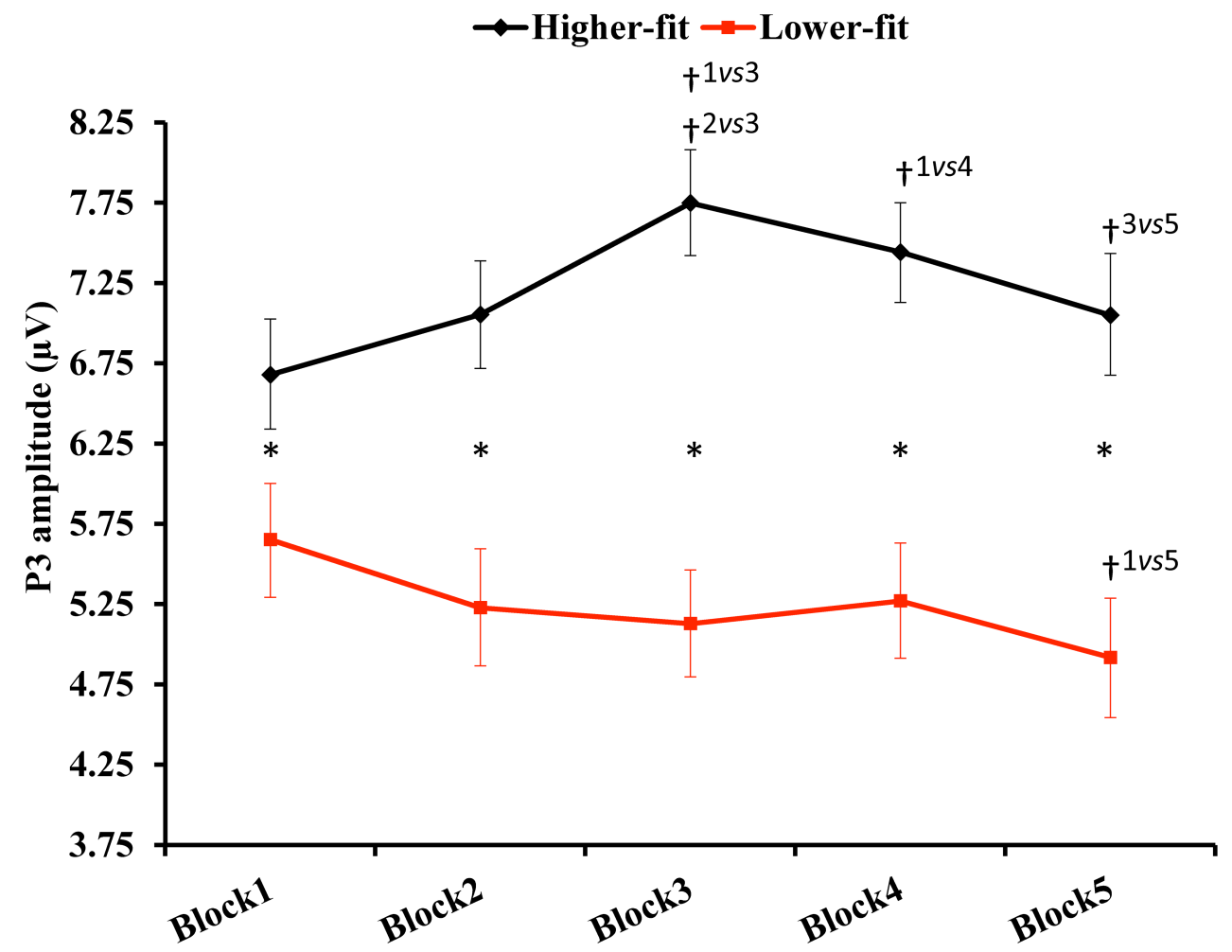




\section{Discussion}

In the present study, we tested the positive relation between aerobic fitness and sustained attention capacity by comparing RT performance, the CNV and the P3 amplitude, in a 60minute attention demanding task of two groups of participants: higher- and lower-fit young adults.

The results showed that higher-fit participants responded faster than lower-fit participants during the first three blocks of the task (i.e., 36 minutes). This was accompanied by larger CNV amplitude in the same blocks in higher-fit than in lower-fit adults; however, this difference disappeared in the later blocks. Crucially, higher-fit participants maintained larger P3 amplitude throughout the task compared to lower-fit, who showed a reduction in the P3 magnitude as a function of the time-on-task.

Concerning performance in the PVT, the results in the first part of the task are in accordance with the previous study by Luque-Casado et al. (27), suggesting better vigilance capacity in higher-fit young adults relative to their lower-fit counterparts. However, a greater vigilance decrement was shown in higher-fit than in lower-fit participants. The ERP data provided crucial information in order to understand these apparently contradictory results. The CNV and RT patterns were closely related, such that the higher-fit group showed larger CNV amplitude in the first half of the task (blocks 1 to 3) compared to the lower-fit group, but, again, these differences disappeared in the later blocks. It is known that temporal preparation substantially enhances performance by reducing response times to an imminent signal in simple RT tasks (18) and, in fact, the magnitude of the CNV has been shown to depend on sustained attention (4). Thus, the improved performance in higher-fit participants indexed by shorter RTs in the early blocks of the task might be the result of better cue facilitation despite the high temporal uncertainty of the task, suggesting an enhanced topdown processing in terms of endogenous preparation in this group. This supports previous 
evidence showing that higher-fit participants are better at the stage of preparation prior to target onset and the behavioral response $(19,35)$ as well as activating and adapting neural processes involved in cognitive control to meet and maintain task goals (7). However, important here is that the response preparation benefit could not be maintained throughout the task by higher-fit participants and disappeared over time, leading in turn to the loss of group differences in RTs.

Maintaining attention for long periods of time requires hard mental work leading to a mental fatigue state (38), which has been evidenced in our study by the vigilance decrement over time in both groups. Additionally, it is known that mental fatigue results in a reduction of top-down attentional capacity (5). Therefore, even though both groups were affected by mental fatigue, in the case of higher-fit participants it appeared to impact on the enhanced endogenous preparation as a function of the time-on-task, thus leading to the disappearance of the improved behavioral performance. Indeed, this would agree with previous studies showing greater difficulties in maintaining the state of endogenous preparation in mentally fatigued participants (25) evidenced by the significant attenuation of brain activity elicited by cue information (i.e., CNV) as a function of time-on-task (24).

In general terms, the P3 potential is thought to reflect the amount of attentional resources directed to task-relevant information in the stimulus environment (29) and accordingly, the P3 should be taken as the relevant index of sustained attention in our study. In accordance with previous research (15), we observed larger P3 amplitude for the high-fit participants suggesting an enhanced ability to allocate attentional resources relative to their lower-fit counterparts. Novel to these previous accounts is the fact that, first, we measured directly and specifically the ability to maintain attention, unlike previous studies whose interest was focused mainly on investigating the relationship of fitness with cognitive control; and second, the time-on-task effect differentially affected P3 amplitude in higher-fit and lower-fit 
participants showing a depletion in the allocation of attentional resources from the beginning of the task only in the latter group.

Interestingly, higher-fit participants maintained larger P3 amplitude relative to lower-fit participants, and demonstrated maximum amplitude in the third temporal block. The amplitude significantly decreased through the end of the task following the third block, although, importantly, never reaching lower values than in the first block. This apparent depletion in attentional resources allocation coincided in time with the disappearance of their improved temporal preparation (indexed by the CNV), which could have led indirectly to an increase in demands for maintaining the task goal in the absence of cue facilitation, causing added mental fatigue and leading to the observed decrease in P3 amplitude from the peak reached in block 3. In any case, it is noteworthy that the higher-fit group always showed greater amplitude of P3 relative to the lower-fit group throughout the task, and crucially, only the lower-fit group showed a significant reduction of the P3 amplitude from the beginning of the task. All in all, and according to previous evidence (20), these results can be taken as an index of enhanced ability to maintain the allocation of attentional resources over time in higher-fit participants with respect to lower-fit participants.

In conclusion, higher fitness was related to neuroelectric activity suggestive of better overall sustained attention and a better response preparation (although only in the first part of the task). Taken together, the current dataset replicates and extends this area of research by demonstrating an association between higher amounts of aerobic fitness and sustained attention. However, it is important to consider that sport training context is a stimulating environment where both cardiovascular fitness and perceptual-cognitive skills are enhanced, which might in turn influence cognitive function. Consequently, other factors in addition to fitness might also account for (at least part) of the group differences reported here. Hence, future research would benefit from study designs that include specific sport groups and 
account for the potential influence of the perceptual-cognitive skills involved in sport training context to clarify the specific, rather than combined, effect both of the cardiovascular fitness and the sport training context on vigilance performance. Finally, since sustaining attention is a basic requirement for information processing and, consequently, a fundamental component of the general cognitive capacities of humans, our findings provide additional evidence of the broad relevance for public health of a physically active lifestyle aimed at improving aerobic fitness. In effect, this should be considered in environments such as education (i.e., in integrated educational development plans), or many other aspects of everyday life and professional activities (e.g., driving, surgery, military and border surveillance, lifeguarding or air traffic control), since this might lead to a reduction of the likelihood of attentional failures in prolonged high demands environments.

\section{Acknowledgments}

This research was supported by a predoctoral grant from the Spanish Ministerio de Educación, Cultura y Deporte (FPU-AP2010-3630) to the first author, and research grants from the Ministerio de Economía y Competitividad (PSI2013-46385-P) and the Junta de Andalucía (SEJ-6414) to Daniel Sanabria. The funders had no role in study design, data collection and analysis, decision to publish, or preparation of the manuscript. We thank to Enrique Molina for providing his knowledge and assistance in the statistical data analyses, and to all the participants who took part in the experiment. We also thank to Human Psychophysiology and Health Research Group (University of Granada) for allowing us to use their facilities and assessment instruments. No conflicting financial, consultant, institutional, or other interests exist. Results of the present study do not constitute endorsement by the American College of Sports Medicine.

\section{References}

1. Arito H, Oguri M. Contingent negative variation and reaction time of physicallytrained subjects in simple and discriminative tasks. Ind Health 1990;28(2):97-106. 
2. Ballester R, Huertas F, Yuste FJ, Llorens F, Sanabria D. The Relationship between Regular Sports Participation and Vigilance in Male and Female Adolescents. PLoS ONE 2015;10(4):e0123898.

3. Basner M, Dinges DF. Maximizing Sensitivity of the Psychomotor Vigilance Test (PVT) to Sleep Loss. Sleep 2011;34(5):581-91.

4. Bickel S, Dias EC, Epstein ML, Javitt DC. Expectancy-related modulations of neural oscillations in continuous performance tasks. Neuroimage 2012;62(3):1867-76.

5. Boksem MAS, Meijman TF, Lorist MM. Effects of mental fatigue on attention: An ERP study. Cognitive Brain Research 2005;25(1):107-16.

6. Bunce D. Age differences in vigilance as a function of health-related physical fitness and task demands. Neuropsychologia 2001;39(8):787-97.

7. Chaddock L, Erickson KI, Prakash RS, et al. A functional MRI investigation of the association between childhood aerobic fitness and neurocognitive control. Biol Psychol 2012;89(1):260-8.

8. Davies DR, Parasuraman R. The psychology of vigilance. Academic Press; 1982.306

9. Davis JA, Vodak P, Wilmore JH, Vodak J, Kurtz P. Anaerobic threshold and maximal aerobic power for three modes of exercise. J Appl Physiol 1976;41(4):544-50.

10. Delorme A, Makeig S. EEGLAB: an open source toolbox for analysis of single-trial EEG dynamics including independent component analysis. Journal of Neuroscience Methods 2004;134(1):9-21.

11. Ernst MD. Permutation Methods: A Basis for Exact Inference. Statist. Sci. 2004;19(4):676-85.

12. Good PI. Permutation, Parametric, and Bootstrap Tests of Hypotheses. Springer Science \& Business Media; 2006.331

13. Grier RA, Warm JS, Dember WN, et al. The Vigilance Decrement Reflects Limitations in Effortful Attention, Not Mindlessness. Human Factors: The Journal of the Human Factors and Ergonomics Society 2003;45(3):349-59.

14. Guiney H, Machado L. Benefits of regular aerobic exercise for executive functioning in healthy populations. Psychon Bull Rev 2013;20(1):73-86.

15. Hillman $\mathrm{CH}$, Buck SM, Themanson JR, Pontifex MB, Castelli DM. Aerobic fitness and cognitive development: Event-related brain potential and task performance indices of executive control in preadolescent children. Dev Psychol 2009;45(1):114-29.

16. Hillman $\mathrm{CH}$, Erickson KI, Kramer AF. Be smart, exercise your heart: exercise effects on brain and cognition. Nature Reviews Neuroscience 2008;9(1):58-65. 
17. Hoffmann S, Falkenstein M. The Correction of Eye Blink Artefacts in the EEG: A Comparison of Two Prominent Methods. PLoS ONE 2008;3(8):e3004.

18. Jennings JR, van der Molen MW. Preparation for speeded action as a psychophysiological concept. Psychol Bull 2005;131(3):434-59.

19. Kamijo K, Pontifex MB, O'Leary KC, et al. The effects of an afterschool physical activity program on working memory in preadolescent children. Dev Sci 2011;14(5):104658.

20. Koelega HS, Verbaten MN, van Leeuwen TH, Kenemans JL, Kemner C, Sjouw W. Time effects on event-related brain potentials and vigilance performance. Biological Psychology 1992;34(1):59-86.

21. Kwan MY, Cairney J, Faulkner GE, Pullenayegum EE. Physical activity and other health-risk behaviors during the transition into early adulthood: a longitudinal cohort study. Am J Prev Med 2012;42(1):14-20.

22. Larue GS, Rakotonirainy A, Pettitt AN. Driving performance impairments due to hypovigilance on monotonous roads. Accid Anal Prev 2011;43(6):2037-46.

23. Lopez-Calderon J, Luck SJ. ERPLAB: an open-source toolbox for the analysis of event-related potentials. Front Hum Neurosci [Internet] 2014 [cited 2015 Sep 16]; 8 (213). Available from: http://journal.frontiersin.org/article/10.3389/fnhum.2014.00213/full. doi:10.3389/fnhum.2014.00213

24. Lorist MM. Impact of top-down control during mental fatigue. Brain Research 2008; 1232:113-23.

25. Lorist MM, Klein M, Nieuwenhuis S, De Jong R, Mulder G, Meijman TF. Mental fatigue and task control: planning and preparation. Psychophysiology 2000;37(5):614-25.

26. Luck SJ. An Introduction to the Event-Related Potential Technique. Cambridge, Mass: The Mit Press; 2005.376

27. Luque-Casado A, Zabala M, Morales E, Mateo-March M, Sanabria D. Cognitive Performance and Heart Rate Variability: The Influence of Fitness Level. PLoS ONE 2013;8(2):e56935.

28. Pesarin F, Salmaso L. The permutation testing approach: a review. Statistica 2010;70(4):481-509.

29. Polich J, Kok A. Cognitive and biological determinants of P300: an integrative review. Biological Psychology 1995;41(2):103-46.

30. Pontifex MB, Hillman CH, Polich J. Age, physical fitness, and attention. Psychophysiology 2009;46(2):379-87. 
31. Pontifex MB, Scudder MR, Drollette ES, Hillman CH. Fit and vigilant: the relationship between poorer aerobic fitness and failures in sustained attention during preadolescence. Neuropsychology 2012;26(4):407-13.

32. Salthouse TA, Davis HP. Organization of cognitive abilities and neuropsychological variables across the lifespan. Developmental Review 2006;26(1):31-54.

33. Sarter M, Givens B, Bruno JP. The cognitive neuroscience of sustained attention: where top-down meets bottom-up. Brain Research Reviews 2001;35(2):146-60.

34. Smith PJ, Blumenthal JA, Hoffman BM, et al. Aerobic Exercise and Neurocognitive Performance: A Meta-Analytic Review of Randomized Controlled Trials. Psychosom Med 2010;72(3):239-52.

35. Stroth S, Kubesch S, Dieterle K, Ruchsow M, Heim R, Kiefer M. Physical fitness, but not acute exercise modulates event-related potential indices for executive control in healthy adolescents. Brain Research 2009;1269:114-24.

36. Trillenberg P, Verleger R, Wascher E, Wauschkuhn B, Wessel K. CNV and temporal uncertainty with "ageing" and "non-ageing" S1-S2 intervals. Clinical Neurophysiology 2000;111(7):1216-26.

37. Walter WG, Cooper R, Aldridge VJ, McCallum WC, Winter AL. Contingent Negative Variation : An Electric Sign of Sensori-Motor Association and Expectancy in the Human Brain. Nature 1964;203(4943):380-4.

38. Warm JS, Parasuraman R, Matthews G. Vigilance Requires Hard Mental Work and Is Stressful. Human Factors: The Journal of the Human Factors and Ergonomics Society 2008;50(3):433-41.

39. Wilkinson RT, Houghton D. Field test of arousal: a portable reaction timer with data storage. Hum Factors 1982;24(4):487-93.

40. Yeh MP, Gardner RM, Adams TD, Yanowitz FG, Crapo RO. "Anaerobic threshold": problems of determination and validation. J Appl Physiol Respir Environ Exerc Physiol 1983;55(4):1178-86. 